\title{
Long-term chorioretinal changes after photodynamic therapy for chronic central serous chorioretinopathy
}

\author{
Helena Vasconcelos • Inês Marques • A. Rita Santos • \\ Pedro Melo • Isabel Pires • João Figueira • \\ José Faria de Abreu • M. Luz Cachulo • Rufino Silva
}

Received: 29 October 2012 /Revised: 24 November 2012 / Accepted: 15 January 2013 / Published online: 7 February 2013

(C) Springer-Verlag Berlin Heidelberg 2013

\begin{abstract}
Purpose To evaluate morphological and functional chorioretinal changes 5 years after standard photodynamic therapy (PDT) for chronic central serous chorioretinopathy (CSC). Methods A retrospective, nonrandomized study, including patients with chronic CSC treated with standard PDT and followed for at least 60 months. All patients underwent a complete ophthalmological examination, and the location and number of treatments were registered. Five or more years after treatment, subfoveal and non-subfoveal treated areas were evaluated with Spectralis optical coherence tomography and microperimetry.

Results Seventeen eyes of 15 patients were included, with mean age of $48.3 \pm 8.4$ years and a mean follow-up of $80.6 \pm$ 12.4 months (range from 62 to 104 months). All eyes had neurosensory detachment (NSD) at baseline. Treatment was performed under the fovea in $58.8 \%$ and in a non-foveal area in $41.2 \%$ of the eyes. At the final visit all eyes had resolution of the NSD, with a statistical significant reduction in central macular thickness $(p=0.005)$ and preserved neuroretinal thickness $(p=0.839)$. There was a statistical difference between initial and final BCVA $(p<0.001)$ and a mean gain of $8.4 \pm 7.8$ letters. Subfoveal morphological changes in
\end{abstract}

H. Vasconcelos $(\bowtie) \cdot$ I. Marques · I. Pires · J. Figueira •

M. L. Cachulo $\cdot$ R. Silva

Ophthalmology Unit, Centro Hospitalar e Universitário

de Coimbra, Portugal (CHUC), Praceta Mota Pinto,

3000-075 Coimbra, Portugal

e-mail: hvasconcelos@gmail.com

A. R. Santos • P. Melo - I. Pires · J. Figueira - J. F. de Abreu •

M. L. Cachulo $\cdot$ R. Silva

Association for Innovation and Biomedical Research on Light

and Image, Coimbra, Portugal (AIBILI), Coimbra, Portugal

J. Figueira $\cdot$ J. F. de Abreu $\cdot$ R. Silva

Faculty of Medicine, University of Coimbra,

Portugal (FMUC), Coimbra, Portugal external limiting membrane (ELM) and in photoreceptor inner and outer segment junction (IS/OS) were correlated with final $\operatorname{BCVA}(p=0.015$ and $p=0.014$ respectively), but not with the variation of BCVA. There was a statistical correlation between morphological changes in IS/OS line and retinal sensitivity in the central $12^{\circ}$ and $2^{\circ}(p=0.003$ and $p=0.002$ respectively). The morphological changes in the subfoveal layers were not dependent on treatment location ( $p=0.154, p=0.644$, and $p=1.0$ for ELM, IS/OS line, and retinal pigment epithelium respectively). Subfoveal final mean choroidal thickness was $295.1 \pm 68.7 \mu \mathrm{m}$, and showed no statistical difference from the normal population $(p=0.633)$.

Conclusions Morphological and functional chorioretinal changes, observed 5 or more years after standard PDT for chronic CSC, were not correlated with the location of treatment, neither with the progression of visual acuity or with the location of treatment, and are more likely to be related to the disease itself than with the treatment provided.

Keywords Central serous chorioretinopathy · Photodynamic therapy $\cdot$ Retinal layers morphology $\cdot$ Retinal sensitivity ·

Choroidal thickness

\section{Introduction}

The pathogenesis of central serous chorioretinopathy (CSC) is poorly understood, but choroidal hyperpermeability leading to increased permeability of the retinal pigment epithelium (RPE) and accumulation of subretinal fluid (SRF) has been implicated [1,2]. The high rate of spontaneous resolution provides that conservative management can be adopted as first-line treatment in most cases [3]. Photodynamic therapy (PDT) with verteporfin has shown good results, both anatomic and functional, in treating chronic CSC, with either standard or reduced fluence $\left(600 \mathrm{~mW} / \mathrm{cm}^{2}, 300 \mathrm{~mW} / \mathrm{cm}^{2}\right.$ respectively $)$ 
and with either standard or half dose $\left(6 \mathrm{mg} / \mathrm{m}^{2}\right.$ or $3 \mathrm{mg} / \mathrm{m}^{2}$ respectively) [4-16]. However, it has been reported that PDT can be associated with potential side-effects, such as RPE atrophy, neuroretinal thickness (NRT) thinning, development of choroidal neovascularization (CNV), and reduction in macular function [15-19]. All of these complications were reported in short follow-up studies, and PDT long-term effects in retinal morphology and function are still unknown.

The purpose of this study is to characterize functional and morphological chorioretinal changes 5 years after standard PDT for chronic CSC.

\section{Material and methods}

A retrospective, observational, nonrandomized study was performed in 17 eyes of 15 patients with chronic CSC, 5 or more years after standard PDT. At the final visit, all eyes underwent Spectralis optical coherence tomography (OCT) and microperimetry for morphological and functional chorioretinal evaluation.

Chronic CSC was defined as diffuse or poorly defined widespread areas of leakage in fluorescein angiography (FA), from broad areas of RPE damage. Inclusion criteria were: (1) chronic CSC diagnosed by FA and confirmed by indocyanine green angiography (ICGA) and OCT, (2) persistent CSC longer than 6 months of duration or RPE changes induced by multifocal recurrent detachment associated with symptoms of chronic CSC lasting at least 6 months, (3) more than 5 years of follow-up, (4) patient with 18 years or older, (5 absence of spontaneous resolution or improvement induced by empirical treatment such as acetazolamide or ketoconazole, or (6) presence of written informed consent for all patients. Eyes were excluded if additional diagnoses that could compromise visual acuity were present, if previous treatment with laser photocoagulation was performed, or if hypersensitivity to fluorescein or any other condition that might contraindicate PDT or angiographic exams were present.

At the baseline, clinical assessment included best-corrected visual acuity (BCVA) with Early Treatment of Diabetic Retinopathy (ETDRS) chart, slit-lamp biomicroscopy with a contact lens or a noncontact lens, indirect ophthalmoscopy, FA (TRC-50IA, Topcon, Tokyo, Japan), ICGA (TRC-50IA, Topcon, Tokyo, Japan), and OCT (OCT Stratus 3-Carl Zeiss Meditec, Dublin, CA, USA). The Stratus OCT was performed to measure initial and final NRT (markers were placed between the internal limiting membrane and the anterior limit of the SRF, on the smaller thickness of the retina) and central macular thickness (markers were placed between the internal limiting membrane and the RPE, without including it).

All patients were treated with verteporfin (Visudyne, Novartis AG, Basel, Switzerland) ICGA-guided PDT using standard treatment parameters, according to the standard protocol for CNV secondary to age-related macular degeneration (treatment of age-related macular degeneration (TAP) Study Group 1999) [20]. The verteporfin was infused $\left(6 \mathrm{mg} / \mathrm{m}^{2}\right)$ for $10 \mathrm{~min}$ followed by delivery of an activating light dose of $50 \mathrm{~J} / \mathrm{cm}^{2}$ from a $689 \mathrm{~nm}$ laser system (Carl Zeiss, Dublin, CA, USA) with an exposure time of $83 \mathrm{~s}$. The laser spot size for the PDT was the diameter of the choroidal hyperpermeability area, indentified in the ICGA, plus $1 \mathrm{~mm}$. The number of treatments and the size of the spot were registered. The treatment location was also registered as foveal (less than $500 \mu \mathrm{m}$ from the center of the foveal avascular zone) or non-foveal (more than $500 \mu \mathrm{m}$ from the center of the foveal avascular zone).

Evaluations were performed each 3 months in the first year, each 6 months in the secondyear and annually after the secondyear. BCVA and OCT were performed in all visits. FA and ICGA were performed when necessary. Patients were re-treated based on clinical evidence of reappearance or persistence of neurosensory detachment (NSD), showed on OCT.

Improvement in BCVA was considered when a gain of 5 or more letters was achieved, and visual loss when there was a decrease of at least 5 letters in relation to initial BCVA.

At the final visit, Spectralis OCT (Spectralis Heidelberg Engineering Inc., Heidelberg, Germany) was used to evaluate retinal morphology of the subfoveal and treated areas (when non-coincident), to determine the presence of SRF and to measure treated retinal thickness, using the attached measuring software in the Spectralis OCT. Treated retinal thickness was defined as the smallest thickness in the central $500 \mu \mathrm{m}$ in diameter of the treated retina. The study of the morphology of the outer retinal layers was performed at the level of the central macula and treated retina, and included assessment of the external limiting membrane (ELM), photoreceptor inner and outer segment junction (IS/OS), and RPE. ELM was classified as normal or absent; IS/OS line was classified as normal, with interruptions, or absent; RPE was classified as normal or with atrophy. The layers were defined as normal when they were detected as a continuous line, without any defect. The layers were classified with interruptions when they presented with point defects less than $200 \mu \mathrm{m}$ within the studied area, and were classified as absent if there was a discontinuity of more than $200 \mu \mathrm{m}$ in the studied layer. An atrophic layer was referred to a diffuse loss of tissue in a present line.

At the final visit macular microperimetry was also performed, using microperimeter-1 (MP-1) software (Niked, Vigonza, Italy). The MP-1 provides a $45^{\circ}$ view of the retina with automated correction for eye movements. Goldmann III stimuli with a white background and a 4-2 threshold strategy were used. A circular test grid with 45 stimulus points covering an area of $12^{\circ}$ in diameter was applied. The mean retinal sensitivity (RS) within the central $2^{\circ}$ and $12^{\circ}$ in diameter was calculated. The microperimetry sensitivity was also measured in the central $500 \mu \mathrm{m}$ of the treated retina. 
At the final visit, choroidal thickness (CT) was measured with enhanced depth imaging spectral-domain optical coherence tomography (EDI OCT) by positioning the Spectralis OCT close enough to the eye to obtain an inverted image. Horizontal sections of $9 \mathrm{~mm}$, which contain 100 average scans, were obtained and the CT was manually measured using the contained Heidelberg software. CT was defined as the vertical distance from the hyperreflective line of Bruch's membrane to the innermost hyperreflective line of the chorioscleral interface. The central subfoveal CT of both eyes and the CT below the smallest thickness of treated retina of the affected eye were registered. Data from a pilot study reporting the $\mathrm{CT}$ of normal eyes, that were measured using the same techniques, in a population with similar age to our study, were used to compare with our results [21].

For statistical analysis, SPSS software version 20.0 (SPSS Inc, Chicago, IL, USA) was used. The level of statistical significance was calculated with Kruskal-Wallis test, MannWhitney $U$-test, Fisher's exact test, paired samples test, independent sample test and one-sample test depending on the type and distributions of the samples. The statistic correlations were calculated using the Pearson correlation or the Spearman's rho correlation. A $P$ value less than 0.05 or 0.01 , according to the sample and test performed, was considered statistically significant. Written informed consent was obtained from all subjects, and the study was conducted in accordance with the tenets of the Declaration of Helsinki and after institutional review board approval.

\section{Results}

Table 1 summarizes the clinical characteristics of the patients and treatment results. Seventeen eyes of 15 patients with chronic CSC treated with standard dose PDT with verteporfin were included, with a mean follow-up of $80.6 \pm 12.4$ months (range from 62 to 104 months).

ICGA-guided PDT was applied at the foveal area in ten eyes $(58.8 \%)$ and in non-foveal area in seven eyes $(41.2 \%)$, with a mean spot size of $3326.5 \pm 1061.9 \mu \mathrm{m}$ (range from $1,200$ to $5,400 \mu \mathrm{m})$.

The initial mean BCVA was $62.5 \pm 19.6$ letters (Snellen equivalent $20 / 63^{+3}$ ), which ranged from 10 to 85 letters, and the final mean BCVA was $70.9 \pm 20.4$ letters (Snellen equivalent $20 / 40^{+1}$ ), which ranged from 20 to 90 letters. A statistically significant improvement in BCVA $(p<0.001$, paired samples test) was registered, with a mean gain of $8.4 \pm 7.8$ letters (range from loss of two letters to gain of 25 letters). Figure 1 shows the evolution of BCVA during the follow-up. There was also a statistically positive correlation between initial and final BCVA ( $R$ Pearson $=0.925$ and Spearman's rho $=0.752, p<0.001)$. At the final visit, 12 eyes $(70.6 \%$ ) improved vision (gain of 5 or more letters) and five eyes (29.4\%) remained unchanged.
There was no statistical difference in final BCVA between eyes that were treated in the foveal or in the non-foveal area ( $p=0.583$, independent samples test).

During the follow-up, the mean number of treatments was $1.1 \pm 0.3$. Of the two eyes that underwent two sessions of treatment, one was re-treated after 2 years and the other was re-treated after 7 years of the first treatment. In these two eyes, the re-treatment was performed in the same area and for the same leakage spots as the primary PDT.

At the baseline, all eyes had NSD and no eyes had intraretinal fluid. The mean CMT was $257.9 \pm 128.4 \mu \mathrm{m}$ (74$597 \mu \mathrm{m})$ and the mean central NRT was $160.1 \pm 57.4 \mu \mathrm{m}$ (49-290 $\mu \mathrm{m})$. There was a statistical difference between initial and final CMT ( $p=0.005$, paired samples test) which decreased to $176.2 \pm 60 \mu \mathrm{m}(40-318 \mu \mathrm{m})$. However, NRT remained stable during the 5 or more years after PDT, which ranged from $160.1 \pm 57.4 \mu \mathrm{m}$ to $159.2 \pm 56.4 \mu \mathrm{m}(p=0.839$, paired samples test). Final CMT and NRT were statistically correlated with final BCVA (R Pearson=0.80, $p<0.001 ; R$ Pearson $=0.76, p<0.001$; respectively).

At the final visit, we studied the morphology of the outer retinal layers, at subfoveal area and at the treated retina, with Spectralis OCT, and correlated them with final BCVA. Table 2 summarizes the morphological changes found 5 years after PDT, and Table 3 shows the correlation with final BCVA. In the subfoveal area, ELM was normal in 15 eyes ( $88.2 \%$ ) and absent in two eyes $(11.8 \%)$. In these two eyes, baseline Stratus OCT already showed retinal atrophy (NRT of 49 and $82 \mu \mathrm{m})$. Morphological changes found in subfoveal ELM were correlated with final BCVA ( $p=0.015$, Mann-Whitney $U$ test), but showed no correlation with the variation of BCVA ( $p=1.0$, Mann-Whitney $U$ test). Subfoveal IS/OS line was classified as normal in six eyes (35.3\%), with interruptions in eight eyes (47.1\%) and as absent in three eyes (17.6\%). There was also a statistical correlation between IS/OS line morphology and final BCVA ( $p=0.014$, Kruskal-Wallis test), but the difference in BCVA was only statistically significant between the normal and absent groups ( $p=0.011$, Kruskal-Wallis test). As it was with the ELM, the alterations found in IS/OS line did not correlate with the variation of BCVA ( $p=0.507$, KruskalWallis test). In relation to RPE, the morphology of the layers was classified as normal in five eyes (29.4\%) and with atrophy in 12 eyes (70.6 \%). There was no statistical correlation between the RPE morphology and final or variation of BCVA ( $p=0.064$ and $p=0.646$ respectively; Mann-Whitney $U$ test). Only two eyes in this study had a final BCVA less than 30 letters, and both eyes improved vision after treatment (case 3 and 4). Spectralis OCT in these eyes showed an absent ELM and IS/OS line and RPE atrophy. Baseline Stratus OCT already showed retinal atrophy.

Final morphological changes described above were present in the fovea, independent on treatment location, whether it was performed in the foveal or in the non-foveal area $(p=0.154$, 
Table 1 Clinical changes in BCVA, central macular thickness, neuroretinal thickness and choroidal thickness

\begin{tabular}{|c|c|c|c|c|c|c|c|c|c|c|c|c|c|c|}
\hline \multirow{2}{*}{$\begin{array}{l}\text { Patient } \\
\text { no. }\end{array}$} & \multirow[t]{2}{*}{ Gender } & \multirow[t]{2}{*}{ Eye } & \multirow[t]{2}{*}{ Age } & \multirow{2}{*}{$\begin{array}{l}\text { Follow-up } \\
\text { (months) }\end{array}$} & \multicolumn{2}{|c|}{ PDT } & \multicolumn{3}{|l|}{ Baseline } & \multicolumn{5}{|c|}{ Final follow-up } \\
\hline & & & & & No. & Local & $\begin{array}{l}\text { BCVA } \\
\text { (letters) }\end{array}$ & $\begin{array}{l}\text { CMT } \\
(\mu \mathrm{m})\end{array}$ & $\begin{array}{l}\text { NRT } \\
(\mu \mathrm{m})\end{array}$ & $\begin{array}{l}\text { BCVA } \\
\text { (letters) }\end{array}$ & $\begin{array}{l}\text { CMT } \\
(\mu \mathrm{m})\end{array}$ & $\begin{array}{l}\text { NRT } \\
(\mu \mathrm{m})\end{array}$ & $\begin{array}{l}\text { SF CT } \\
(\mu \mathrm{m})\end{array}$ & $\begin{array}{l}\text { PDT CT } \\
(\mu \mathrm{m})\end{array}$ \\
\hline 1 & M & OD & 42 & 76 & 1 & Foveal & 52 & 174 & 157 & 50 & 158 & 143 & 356 & 356 \\
\hline 2 & M & OS & 53 & 73 & 1 & Foveal & 50 & 224 & 182 & 65 & 201 & 182 & 253 & 253 \\
\hline 3 & M & OD & 47 & 104 & 1 & N-foveal & 25 & 74 & 49 & 30 & 40 & 40 & 300 & 100 \\
\hline 4 & M & OS & 47 & 104 & 1 & $\mathrm{~N}$-foveal & 10 & 232 & 82 & 20 & 71 & 62 & 325 & 248 \\
\hline 5 & M & OD & 48 & 84 & 1 & $\mathrm{~N}$-foveal & 70 & 398 & 224 & 90 & 232 & 224 & 330 & 304 \\
\hline 6 & M & OD & 60 & 69 & 1 & Foveal & 65 & 157 & 107 & 65 & 164 & 157 & 232 & 232 \\
\hline 7 & M & OS & 61 & 70 & 1 & Foveal & 78 & 315 & 199 & 85 & 209 & 199 & 201 & 201 \\
\hline 8 & M & OD & 42 & 62 & 1 & Foveal & 80 & 149 & 149 & 90 & 190 & 149 & 284 & 284 \\
\hline 9 & M & OD & 45 & 74 & 1 & Foveal & 70 & 182 & 182 & 79 & 173 & 162 & 246 & 246 \\
\hline 10 & M & OD & 65 & 66 & 2 & $\mathrm{~N}$-foveal & 83 & 456 & 290 & 85 & 318 & 290 & 173 & 124 \\
\hline 11 & M & OD & 45 & 77 & 1 & Foveal & 65 & 257 & 116 & 63 & 141 & 116 & 333 & 333 \\
\hline 12 & $\mathrm{~F}$ & OD & 51 & 77 & 1 & Foveal & 70 & 190 & 124 & 75 & 175 & 155 & 255 & 255 \\
\hline 13 & M & OD & 53 & 88 & 1 & Foveal & 60 & 291 & 141 & 85 & 183 & 141 & 217 & 217 \\
\hline 14 & M & OD & 46 & 92 & 1 & $\mathrm{~N}$-foveal & 70 & 597 & 174 & 79 & 197 & 174 & 397 & 403 \\
\hline 15 & M & OS & 46 & 92 & 2 & $\mathrm{~N}$-foveal & 65 & 157 & 157 & 80 & 165 & 157 & 372 & 310 \\
\hline 16 & M & OD & 32 & 74 & 1 & $\mathrm{~N}$-foveal & 85 & 273 & 165 & 85 & 179 & 165 & 356 & 330 \\
\hline 17 & M & OS & 38 & 88 & 1 & Foveal & 65 & 259 & 224 & 80 & 199 & 190 & 387 & 387 \\
\hline Mean & - & - & 48.3 & 80.6 & 1.1 & - & 62.5 & 257.9 & 160.1 & 70.9 & 176.2 & 159.2 & 295.1 & 269.6 \\
\hline SD & - & - & 8.4 & 12.4 & 0.3 & - & 19.6 & 128.4 & 57.4 & 20.4 & 60 & 56.4 & 68.7 & 83 \\
\hline
\end{tabular}

$M$ male; $F$ female; $S D$ standard deviation; $N$-foveal non-foveal area; $B C V A$ best-corrected visual acuity. $C M T$ central macular thickness; $N R T$ neuroretinal thickness; SF CT subfoveal central choroidal thickness; PDT CF choroidal thickness in the smallest treated retina

$p=0.644$ and $p=1.0$, for ELM, IS/OS line, and RPE respectively; Fisher's exact test).

Spectralis OCT was also used to evaluate morphological changes and retinal thickness in the treated area. The mean treated retinal thickness was $222.8 \pm 78.5 \mu \mathrm{m}$ (range from 141 to $406 \mu \mathrm{m})$. In the smallest thickness of the treated retina, ELM was normal in 12 eyes (70.6 \%) and absent in five eyes $29.4 \%$ ); IS/OS line was normal in four eyes $(23.5 \%)$, interrupted in six eyes $(35.3 \%)$, and absent in seven eyes (41.2\%); RPE was normal in four eyes (23.5\%) and atrophic in 13 eyes (76.5\%).
Table 2 shows the final RS in the central $12^{\circ}$ and $2^{\circ}$ and in the treated retina, and Table 3 shows correlation with morphological changes. The final mean RS within the central $12^{\circ}$ and $2^{\circ}$ were $15.9 \pm 5.1 \mathrm{~dB}$ (range 1.8-19.9 dB) and $14.8 \pm 5.2 \mathrm{~dB}$ (range $2.2-19.7 \mathrm{~dB}$ ) respectively, and were statistically correlated with final BCVA $(R$ Pearson $=0.86$ and $p<0.001$, $R$ Pearson $=0.90$ and $p<0.001$ respectively), but not with the variation of BCVA. The mean microperimetry within the treated retina was $11.7 \pm 6.5 \mathrm{~dB}$ (range $0-19.6 \mathrm{~dB}$ ) and showed no correlation with laser spot PDT size. Central $2^{\circ} \mathrm{RS}$ was correlated with CMT (Pearson=0.87, $p<0.001$; Spearman's
Fig. 1 Evolution of BCVA, showing an improvement of 8.4 letters, after standard PDT, at final follow-up. $B C V A$ bestcorrected visual acuity. $M$ months

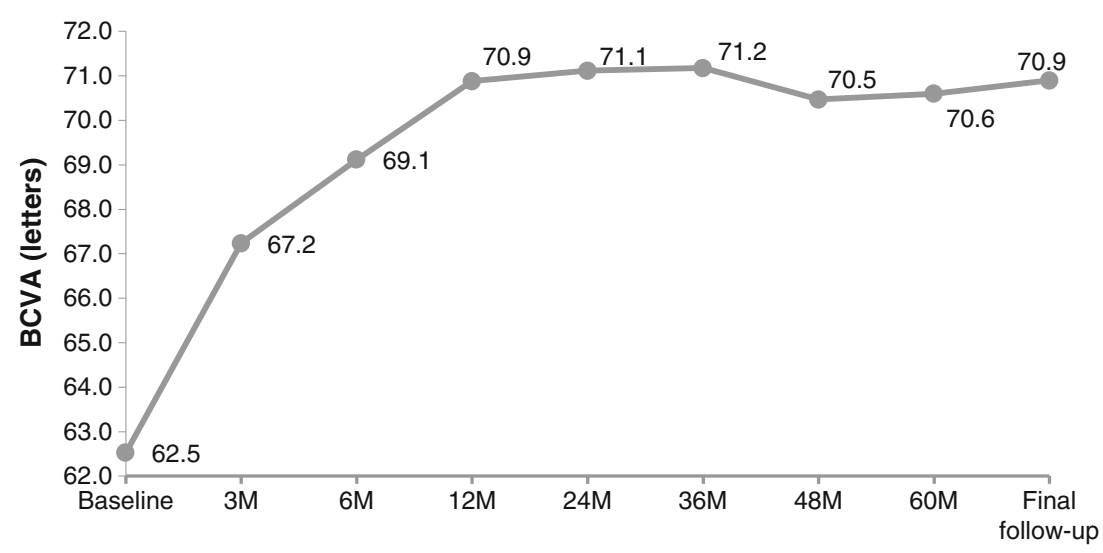


Table 2 Retinal morphological changes in subfoveal and treated retina area; retinal sensitivity in central $12^{\circ}$ and $2^{\circ}$ and in treated retina, at final follow-up

\begin{tabular}{|c|c|c|c|c|c|c|c|c|c|}
\hline \multirow[t]{2}{*}{ Patient no. } & \multicolumn{3}{|c|}{ Subfoveal área } & \multicolumn{3}{|c|}{ Treated retina area } & \multicolumn{3}{|l|}{ MP-1 (dB) } \\
\hline & ELM & IS/OS line & RPE & ELM & IS/OS line & RPE & Central $12^{\circ}$ & Central $2^{\circ}$ & Treated retina \\
\hline 1 & Normal & Interruptions & Atrophy & Normal & Interruptions & Atrophy & 17.13 & 11.15 & 3.20 \\
\hline 2 & Normal & Normal & Atrophy & Normal & Normal & Atrophy & 19.64 & 18.77 & 17.20 \\
\hline 3 & Absent & Absent & Atrophy & Absent & Absent & Atrophy & 1.82 & 2.15 & 0 \\
\hline 4 & Absent & Absent & Atrophy & Absent & Absent & Atrophy & 5.11 & 5.23 & 0 \\
\hline 5 & Normal & Normal & Normal & Absent & Absent & Normal & 19.38 & 19.69 & 16.80 \\
\hline 6 & Normal & Interruptions & Normal & Normal & Interruptions & Normal & 12.18 & 12.92 & 14.00 \\
\hline 7 & Normal & Normal & Normal & Normal & Normal & Normal & 18.93 & 18.00 & 16.40 \\
\hline 8 & Normal & Interruptions & Atrophy & Normal & Interruptions & Atrophy & 16.89 & 18.46 & 17.00 \\
\hline 9 & Normal & Interruptions & Atrophy & Normal & Interruptions & Atrophy & 18.76 & 17.23 & 15.60 \\
\hline 10 & Normal & Normal & Normal & Normal & Normal & Atrophy & 19.87 & 19.54 & 19.60 \\
\hline 11 & Normal & Absent & Atrophy & Normal & Absent & Atrophy & 15.24 & 10.00 & 6.80 \\
\hline 12 & Normal & Interruptions & Atrophy & Normal & Interruptions & Atrophy & 15.98 & 13.31 & 10.60 \\
\hline 13 & Normal & Normal & Normal & Normal & Normal & Normal & 19.47 & 18.77 & 16.40 \\
\hline 14 & Normal & Interruptions & Atrophy & Absent & Absent & Atrophy & 18.36 & 16.77 & 14.60 \\
\hline 15 & Normal & Interruptions & Atrophy & Normal & Absent & Atrophy & 16.04 & 12.95 & 4.40 \\
\hline 16 & Normal & Normal & Atrophy & Absent & Absent & Atrophy & 18.09 & 18.54 & 11.00 \\
\hline 17 & Normal & Interruptions & Atrophy & Normal & Interruptions & Atrophy & 18.18 & 18.54 & 16.00 \\
\hline
\end{tabular}

$M P-1$ retinal sensitivity measured by microperimeter-1; Central $12^{\circ}$ central $12^{\circ}$ retinal sensitivity; Central $2^{\circ}$ central $2^{\circ}$ retinal sensitivity

rho $=0.90, p<0.001)$. In the central $12^{\circ}$ and $2^{\circ}$, RS was statistically correlated with subfoveal morphological changes in ELM ( $p=0.015$, Mann-Whitney $U$ test) and IS/OS line ( $p=0.003$ and $p=0.002$ respectively, Kruskal-Wallis test). Considering IS/OS line changes, RS within the central $12^{\circ}$ and $2^{\circ}$ was better in the normal than in the absent group ( $p=0.003$ and $p=0.002$ respectively, Kruskal-Wallis test). In the treated retina, IS/OS line morphological changes were statistically correlated with $\operatorname{RS}$ ( $p=0.027$, Kruskal-Wallis test) and a statistical difference between the normal and absent group was also registered ( $p=0.022$, Kruskal-Wallis test). There was no statistical correlation between the morphological changes in RPE and RS, in the subfoveal or in the treated retina. There was also no statistical correlation between the morphological changes in ELM and RS in the treated retina.

Representative cases correlating retinal function and morphology are shown (Figs. 2 and 3).

The final subfoveal CT of the treated eyes and was $295.1 \pm$ $68.7 \mu \mathrm{m}$ (range 173-397 $\mu \mathrm{m}$ ), and when compared to a normal population, with a mean age of 52 years, in which the subfoveal $\mathrm{CT}$ is about $287 \mu \mathrm{m}$, there was no statistical difference ( $p=0.633$, one-sample test). Excluding the eyes with active bilateral disease (four eyes), the subfoveal $\mathrm{CT}$ of the treated eyes was $278.7 \pm 67.6 \mu \mathrm{m}$ and showed no difference from the
Table 3 Final mean BCVA and central 12 and $2^{\circ}$ retinal sensitivity according to the subfoveal morphological changes; and mean retinal sensitivity in the treated retina according to morphological changes observed in the retina. Level of statistical significance between morphological changes and BCVA and retinal sensitivity is also shown

\begin{tabular}{|c|c|c|c|c|c|c|c|c|c|c|c|c|}
\hline & \multicolumn{3}{|c|}{ Final BCVA (letters) } & \multicolumn{3}{|c|}{$\operatorname{MP} 12^{\circ}(\mathrm{dB})$} & \multicolumn{3}{|c|}{$\operatorname{MP} 2^{\circ}(\mathrm{dB})$} & \multicolumn{3}{|c|}{ MP PDT (dB) } \\
\hline & ELM & $\begin{array}{l}\text { IS/OS } \\
\text { line }\end{array}$ & RPE & ELM & $\begin{array}{l}\text { IS/OS } \\
\text { line }\end{array}$ & RPE & ELM & $\begin{array}{l}\text { IS/OS } \\
\text { line }\end{array}$ & RPE & ELM & $\begin{array}{l}\text { IS/OS } \\
\text { line }\end{array}$ & RPE \\
\hline Normal & $77.1 \pm 11.5$ & $82.5 \pm 8.8$ & $82 \pm 9.7$ & $17.6 \pm 2.1$ & $19.2 \pm 0.6$ & $18 \pm 3.3$ & $16.3 \pm 3.3$ & $18.9 \pm 0.6$ & $17.8 \pm 2.8$ & $13.1 \pm 5.5$ & $17.5 \pm 1.5$ & $15.9 \pm 1.3$ \\
\hline Interruptions & - & $74.8 \pm 12.1$ & - & - & $16.7 \pm 2.1$ & - & - & $15.2 \pm 2.9$ & - & - & $12.7 \pm 5.2$ & - \\
\hline Atrophy & - & - & $66.3 \pm 22.2$ & - & - & $15.1 \pm 5.6$ & - & - & $13.6 \pm 5.6$ & & & $10.5 \pm 6.9$ \\
\hline Absent & $25 \pm 7.1$ & $37.7 \pm 22.5$ & - & $3.5 \pm 2.3$ & $7.4 \pm 6.9$ & - & $3.7 \pm 2.2$ & $5.8 \pm 3.9$ & - & $8.5 \pm 8$ & $7.7 \pm 6.7$ & - \\
\hline$p$ & 0.015 & 0.014 & 0.064 & 0.015 & 0.003 & 0.082 & 0.015 & 0.002 & 0.064 & 0.078 & 0.027 & 0.245 \\
\hline
\end{tabular}

$E L M$ external limiting membrane. IS/OS photoreceptor inner and outer segment junction. RPE retinal pigment epithelium. $M P 12^{\circ}$ central $12^{\circ}$ retinal sensitivity; $M P 2^{\circ}=$ central $2^{\circ}$ retinal sensitivity: $M P P D T$ retinal sensitivity within the treated retina 


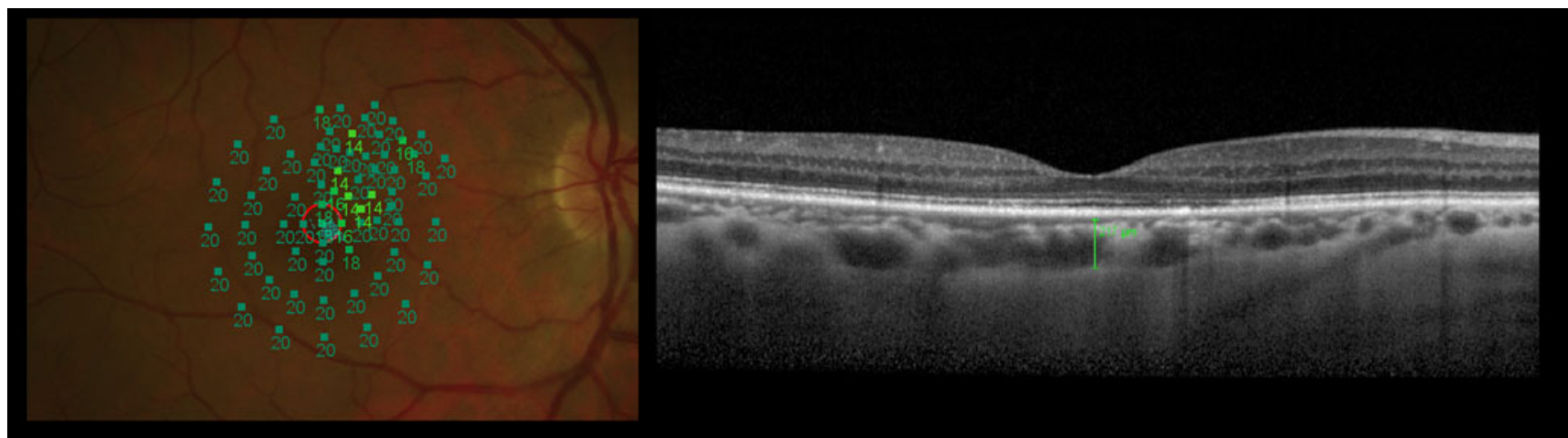

Fig. 2 Case 13: MP-1 showed a mean retinal sensitivity in the central $12^{\circ}$ and $2^{\circ}$ of $19.5 \mathrm{~dB}$ and $18.8 \mathrm{~dB}$ respectively; in the foveal treated retina, mean retinal sensitivity was $16.4 \mathrm{~dB}$. Spectralis OCT showed a subfoveal normal EML, IS/OS line and RPE; central subfoveal choroidal thickness was $217 \mu \mathrm{m}$

subfoveal CT of the unaffected fellow eyes, which was $282.2 \pm$ $60 \mu \mathrm{m}(p=0.867$, paired samples test). The final BCVA showed no statistical correlation with the subfoveal CT, when considering all the eyes or only those which received foveal treatment ( $p=0.526$ and $p=0.256$ respectively, Pearson correlation). Age and subfoveal CT showed a negative correlation $(R$ Pearson $=-0.80, p<0.001$; Spearman's rho $=-0.76$, $p<0.001)$. CT was also measured in the corresponding area of the treated retina, and the mean thickness was $269.6 \pm 83 \mu \mathrm{m}$ (range 100-403 $\mu \mathrm{m}$ ). Subdividing into areas of treatment, the CT was $276.4 \pm 62.3 \mu \mathrm{m}$ (range 201-387 $\mu \mathrm{m}$ ) in the foveal area and $259.9 \pm 111.1 \mu \mathrm{m}$ (range $100-403 \mu \mathrm{m}$ ) in the non-foveal area. Subfoveal CT of the eyes that were treated in the foveal area also showed no statistical difference between the normal population ( $p=0.604$, one-sample test).

\section{Discussion}

In the current study we evaluate the long-term chorioretinal functional and morphological changes 5 or more years after standard PDT for chronic CSC. Several studies have shown favorable results with PDT in treating chronic CSC [4-16]. Our report is the first one to describe the efficacy, the safety, and the chorioretinal changes 5 or more years after standard
PDT for chronic CSC. The presumed therapeutic mechanism of action of PDT is closure of vascular channels in the choriocapillaris, based on the notion that SRF accumulation is caused by choroidal hyperpermeability [22, 23]. However, there are some questions related to the PDT long-term effect in retina. Some authors have described RPE changes after PDT, probably related with hypoxic damage caused by closure of vascular channels in the choriocapillaris [15]. Copete et al. [16] described NRT thinning 1 year after PDT and other authors reported development of CNV and reduction of macular function after PDT [17-19]. Therefore, more recently, some authors suggest alternative therapeutic protocols, reporting a favorable outcome, without retina side-effects, when using reduced fluence or half-dose verteporfin [4-10]. Most of these studies offer no consistent data regarding the benefits of these alternative treatment protocols, mostly because of the small number of patients included and short-term follow-up. Moreover, a direct correlation of these chorioretinal changes with PDT is still lacking.

We evaluated 17 eyes treated with standard verteporfin PDT, in which $58.8 \%$ were treated in the foveal area and $41.2 \%$ in the non-foveal area.

A significant improvement in BCVA was registered, and resolution of the NSD occurred in $100 \%$ of the eyes at the final follow-up. Several different studies have also supported

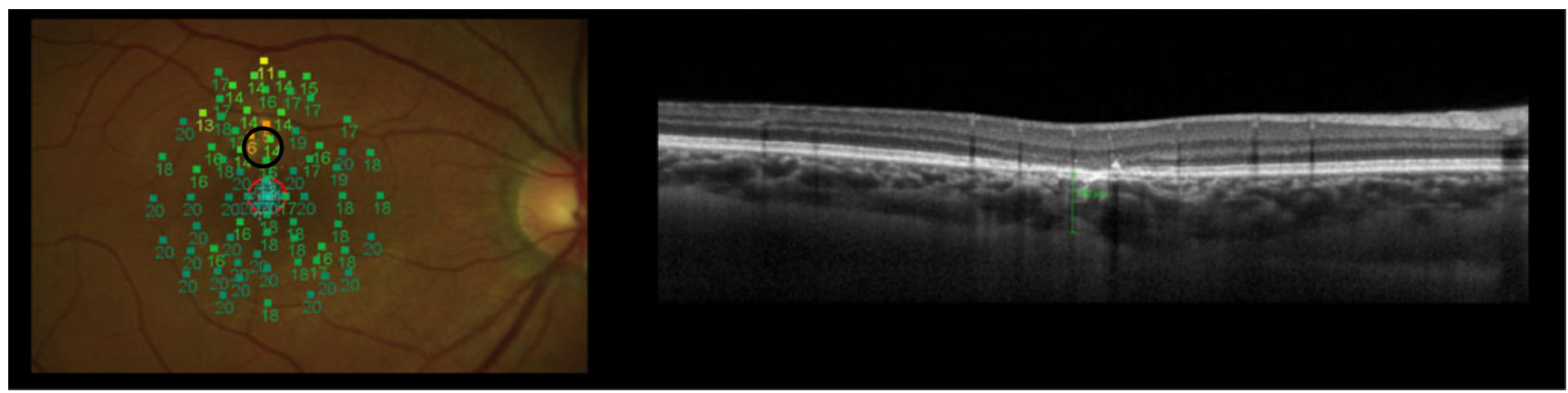

Fig. 3 Case 16: MP-1 showed a mean retinal sensitivity in the central $12^{\circ}$ and $2^{\circ}$ of $18.1 \mathrm{~dB}$ and $18.5 \mathrm{~dB}$ respectively; the dark circle shows the non-foveal treated retina and the mean retinal sensitivity in that area was $11 \mathrm{~dB}$. Spectralis OCT of the smallest treated retina showed an absent ELM and IS/OS line and atrophic RPE; choroidal thickness below the treated retina was $330 \mu \mathrm{m}$ 
the favorable results of standard PDT for chronic CSC [11-16]. Cardillo Piccolino et al. [15] performed standard PDT in 16 eyes with chronic CSC, and reported resolution of NSD in $81 \%$ and BCVA improvement (1-4 lines) in $69 \%$ of the eyes. Also Copete et al. [16] showed complete resolution of NSD in $100 \%$ and improvement of BCVA in $60 \%$ of the eyes, 1 year after standard PDT. A longer follow-up is reported by Silva et al. [24], with a resolution of NSD in $93.4 \%$ and an improvement of BCVA in $74 \%, 4$ years after standard PDT. In the present study, CMT showed a statistically significant reduction, 5 years after standard PDT, corroborating the effectiveness of long-term standard PDT treatment in the resolution of NSD. The neuroretinal thickness remained unchanged during the 5 years of follow-up, demonstrating that treatment with standard PDT did not induced neuroretinal atrophy. Silva et al. [24] also did not find neuroretinal thickness thinning 4 years after standard PDT. These results are different from that reported by Copete et al. [16], which showed neuroretinal thickness thinning 1 year after standard PDT. The mean number of treatments was $1.1 \pm 0.3$, and only two eyes $(11.8 \%)$ underwent two treatment sessions. These numbers of recurrence are inferior to others found in shorter follow-up series, consolidating standard PDT long-term effectiveness [13-15].

In our study, Spectralis CT analysis performed 5 or more years after PDT showed subfoveal morphological changes in ELM and IS/OS line that were statistically correlated with final BCVA. Retinal morphological changes after PDT were also reported in other studies. Cardillo Piccolino et al. [15] reported RPE changes after standard PDT in $38.5 \%$ of eyes, of which $60 \%$ showed RPE atrophy. In another study, Cardillo Piccolino et al. [25] showed that impairment of the photoreceptor layer in CSC was highly correlated with visual loss. In this study, the four eyes that underwent PDT had an atrophic outer photoreceptor layer at baseline, and absent outer photoreceptor layer at final examination [25]. Moon et al. [26] demonstrated that one of the factors limiting visual improvement after PDT for CSC was foveal IS/OS damage. Kim et al. [27] documented that preserved outer photoreceptor layer and continuity of IS/OS line were correlated with better final BCVA. Ooto et al. [28] also showed that visual loss was associated with abnormal cone mosaic patterns and reduced cone densities in eyes with resolved CSC. None of these studies fully demonstrate that the alterations found were caused by the treatment. In our study, the morphological changes found in ELM and IS/OS line were statically correlated with BCVA, but not with the variation of BCVA. Therefore, even eyes with absent ELM or IS/OS line and atrophic RPE were able to improve vision after standard PDT. This means that although eyes with morphological changes in ELM and IS/OS line had the worst BCVA, an improvement in BCVA was achieved with PDT. Another important finding in this study was that morphological changes were not correlated with the area where the treatment was performed, whether it was in the foveal or in the non-foveal area. Therefore, the reported morphological changes in ELM, IS/OS line, and RPE appeared in the foveal area even when the treatment was performed in the non-foveal area. These results show that 5 years after standard PDT, there were morphological changes in the retina, explained, probably, by the natural course of the chronic disease and not induced by the standard PDT treatment.

Visual acuity is the standard way to measure visual function, but it does not describe the full extent of the impact on visual function in patients with compromised central visual fields due to chronic CSC. Microperimetry offers the option to test RS while directly observing the fundus, providing the possibility to correlate subjective or clinical changes with visual function. One of the main symptoms of CSC is relative scotoma induced by lower $\mathrm{RS}$ in the central visual field. Therefore, RS provides further information concerning the visual function after treatment with PDT. The MP-1 microperimeter allows automated functional analysis of the macula, associated with real-time correction of eye movements. Studies with microperimetry in eyes with CSC show significantly lower RS not only at the central but also in the paracentral area of the macula [29-32]. Furthermore, Ozdemir et al. [33] found that eyes with resolved CSC had a statistically significant reduced central RS compared with controls. Although many studies have shown improvement in visual function after PDT [29-32], the improvement in BCVA may not be accompanied by an improvement in central RS. Ehrlich et al. [34] reported a statistically significant difference between the proportions of patients who improved BCVA compared with eyes who improved central RS. In our study, the mean final $\mathrm{RS}$ in the central $12^{\circ}$ and $2^{\circ}$ were correlated with final BCVA, but showed no correlation with the variation of the BCVA. This means that decreased RS is probably more related to retinal changes associated with the disease, than to the effect of PDT, suggesting the absence of treatmentinduced adverse effects. In fact, many studies have reported improvement of RS after PDT [29-32]. Fujita et al. [30] showed improvement in RS within the area of serous retinal detachment, as well as in the central $2^{\circ}$ in diameter, 3 months after half-dose verteporfin PDT. Senturk et al. [31] also reported significant improvement in RS in the central $10^{\circ}$ and $20^{\circ}$ and in the PDT laser spot area after half-dose verteporfin PDT. Although in our study we had no data assessing the RS before PDT, the final central RS is similar to the results found in other studies [29-32]. To explain the decreased RS despite the improvement in visual acuity, we studied the morphological changes in the IS/OS line, in the subfoveal, and in the PDT spot area. We found a positive correlation between RS and morphological changes in the 
IS/OS line, and that sensitivity was better in eyes with normal IS/OS line than in eyes with absent IS/OS line. Fujita et al. [35] demonstrated that there is a significant improvement in macular sensitivity after half-dose PDT in eyes with chronic CSC, and that improvement was correlated with the recovery of the IS/OS line. Ojima et al. [36] also showed that defects in IS/OS line were associated with decreased RS. In their study, irregular RPE were also associated with decreased RS [36]. In the present study, however, the morphological changes in the RPE showed no correlation with the RS. Central RS was also correlated with subfoveal ELM morphological changes; however, there were only two eyes with absent ELM, and these two eyes had retinal atrophy and poor prognosis - therefore we believe that this result is not relevant. Accordingly, decreased RS may reflect photoreceptor dysfunction or photoreceptor cell loss, and preserved visual acuity in eyes with reduced central sensitivity is probably the result of reduced foveal and parafoveal photoreceptor density. This corroborates the fact that the assessment of the BCVA may underestimate visual function after successful PDT treatment, and that patients with active CSC may benefit from early treatment, instead of conservative management, to resolve NSD before chronic irreversible damage has occurred in the IS/OS line.

If CSC is caused by choroidal vascular hyperpermeability, CT may be compromised. To examine this possibility, a method was developed to evaluate $\mathrm{CT}$, which consists in using EDI OCT [37]. Studies with EDI OCT in eyes with CSC, or even unaffected fellow eyes, show that the choroid is much thicker than in normal eyes [38-40]. Imamura et al. [38] reported a mean $\mathrm{CT}$ of $505 \mu \mathrm{m}$ in eyes with CSC, which was statistically greater than in normal eyes. The thickening of the choroid was present in both eyes, even if the manifestations of CSC were present in one eye [38], suggesting the systemic etiology of the disease. Margolis et al. [21] found that the mean subfoveal CT in a normal population, with similar age to those in our study, was $287 \mu \mathrm{m}$. Our results showed that the subfoveal CT 5 years after standard PDT was not different from the thickness of the unaffected fellow eyes, or from the thickness of the normal population. Also, the eyes that were treated in the foveal area had a normal final subfoveal CT. A negative correlation between age and CT was registered, as occurs with the normal population [21]. Therefore, 5 years after successful standard PDT treatment, CT showed normal values. Other studies have reported decrease in CT after PDT; however, the mean subfoveal CT in our study is less than in others with shorter follow-up [41, 42]. Maruko et al. reported a mean subfoveal CT of $330 \pm 103 \mu \mathrm{m}$ and $321 \pm$ $68.6 \mu \mathrm{m} 4$ weeks and 1 year after half-dose verteporfin PDT $[41,42]$. Our results support the long-term effectiveness of standard PDT, which eventually reduced CT and, probably, the choroidal hyperpermeability responsible for the leakage from the level of the RPE.
There are some limitations in our study including the small sample, the retrospective design, the subjective classification of retinal layers, and the limited information prior to PDT, since we used the Stratus OCT at the baseline. However, our functional and anatomical results are important to better understand the role of standard PDT in chronic CSC, a disease without established treatment.

In conclusion, our long-term results showed that morphological and functional chorioretinal changes, observed at least 5 years after standard PDT for chronic CSC, were not correlated with the progression of visual acuity or with the location of treatment, and are more likely to be related to the disease itself than to the treatment provided. However, randomized, prospective studies with larger numbers of patients included are needed to determine the exact role of and the appropriate protocol for photodynamic therapy in patients with chronic CSC.

Financial disclosure The author(s) have no proprietary or commercial interest in any material discussed.

\section{References}

1. Gass JD (1967) Pathogenesis of disciform detachment of the neuroepithelium. Am J Ophthalmol 63(Suppl):1-139

2. Guyer DR, Yannuzzi LA, Slakter JS, Sorenson JA, Ho A, Orlock D (1994) Digital indocyanine green videoangiography of central serous chorioretinopathy. Arch Ophthalmol 112:1057-1562

3. Yannuzzi LA (2010) Central serous chorioretinopathy: a personal perspective. Am J Ophthalmol 149:361-363

4. Lim JW, Kang SE, Kim YT, Chung SE, Lee SW (2011) Comparative study of patients with central serous chorioretinopathy undergoing focal laser photocoagulation or photodynamic therapy. $\mathrm{Br}$ J Ophthalmol 95:514-517

5. Lai TY, Chan WM, Li H, Lai RY, Liu DT, Lam DS (2006) Safety enhanced photodynamic therapy with half dose verteporfin for chronic central serous chorioretinopathy: a short term pilot study. Br J Ophthalmol 90:869-874

6. Koytak A, Erol K, Coskun E, Asik N, Öztürk H, Özertürk Y (2010) Fluorescein angiography-guided photodynamic therapy with half-dose verteporfin for chronic central serous chorioretinopathy. Retina 30:1698-1703

7. Chan WM, Lai TY, Lai RY, Tang EW, Liu DT, Lam DS (2008) Safety enhanced photodynamic therapy for chronic central serous chorioretinopathy: one-year results of a prospective study. Retina 28:85-93

8. Chan WM, Lai TY, Lai RY, Liu DT, Lam DS (2008) Half-dose verteporfin photodynamic therapy for acute central serous chorioretinopathy: one-year results of a randomized controlled trial. Ophthalmology 115:1756-1765

9. Reibaldi M, Cardascia N, Longo A, Furino C, Avitabile T, Faro S, Sanfilippo M, Russo A, Uva MG, Munno F, Cannemi V, Zagari M, Boscia F (2010) Standard-fluence versus low-fluence photodynamic therapy in chronic central serous chorioretinopathy: a nonrandomized clinical trial. Am J Ophthalmol 149:307-315

10. Shin JY, Woo SJ, Yu HG, Park KH (2011) Comparison of efficacy and safety between half-fluence and full-fluence photodynamic therapy for chronic central serous chorioretinopathy. Retina 31:119-126 
11. Taban M, Boyer DS, Thomas EL, Taban M (2004) Chronic central serous chorioretinopathy: photodynamic therapy. Am J Ophthalmol 137:1073-1080

12. Yannuzzi LA, Slakter JS, Gross NE, Spaide RF, Costa DL, Huang SJ, Klancnik JM Jr, Aizman A (2003) Indocyanine green angiographyguided photodynamic therapy for treatment of chronic central serous chorioretinopathy: a pilot study. Retina 23:288-298

13. Ruiz-Moreno JM, Lugo FL, Armadá F, Silva R, Montero JA, Arevalo JF, Arias L, Gómez-Ulla F (2010) Photodynamic therapy for chronic central serous chorioretinopathy. Acta Ophthalmol 88:371-376

14. Tarantola RM, Law JC, Recchia FM, Sternberg P Jr, Agarwal A (2008) Photodynamic therapy as treatment of chronic idiopathic central serous chorioretinopathy. Lasers Surg Med 40:671-675

15. Cardillo Piccolino F, Eandi CM, Ventre L, Rigault de la Longrais RC, Grignolo FM (2003) Photodynamic therapy for chronic central serous chorioretinopathy. Retina 23:752-763

16. Copete S, Ruiz-Moreno JM, Cava C, Montero JA (2012) Retinal thickness changes following photodynamic therapy in chronic central serous chorioretinopathy. Graefes Arch Clin Exp Ophthalmol 250:803-808

17. Chan WM, Lam DS, Lai TY, Tam BS, Liu DT, Chan CK (2003) Choroidal vascular remodelling in central serous chorioretinopathy after indocyanine green guided photodynamic therapy with verteporfin: a novel treatment at the primary disease level. Br J Ophthalmol 87:1453-1458

18. Colucciello M (2006) Choroidal neovascularization complicating photodynamic therapy for central serous retinopathy. Retina 26:239-242

19. Lai TY, Chan WM, Lam DS (2004) Transient reduction in retinal function revealed by multifocal electroretinogram following photodynamic therapy. Am J Ophthalmol 137:826-833

20. Treatment of Age-Related Macular Degeneration with Photodynamic Therapy (TAP) Study Group (1999) Photodynamic therapy of subfoveal choroidal neovascularization in age-related macular degeneration with verteporfin: one-year results of 2 randomized clinical trials - TAP report. Arch Ophthalmol 117:1329-1345

21. Margolis R, Spaide RF (2009) A pilot study of enhanced depth imaging optical coherence tomography of the choroid in normal eyes. Am J Ophthalmol 147:811-815

22. Schmidt-Erfurth U, Hasan T, Gragoudas E, Michaud N, Flotte TJ, Birngruber R (1994) Vascular targeting in photodynamic occlusion of subretinal vessels. Ophthalmology 101:1953-1961

23. Schmidt-Erfurth U, Hasan T (2000) Mechanisms of action of photodynamic therapy with verteporfin for the treatment of agerelated macular degeneration. Surv Ophthalmol 45:195-214

24. Silva R, Ruiz-Moreno JM, Gomez-Ulla F, Montero JA, Gregório T, Cachulo ML, Pires I, Cunha-Vaz JG, Murta JN (2013) Photodynamic therapy for chronic central serous chorioretinopathy: a 4 year follow-up study. Retina 33(2):309-315

25. Cardillo Piccolino F, Rigault de la Longrais RC, Ravera G, Eandi CM, Ventre L, Abdollahi A, Manea M (2005) The foveal photoreceptor layer and visual acuity loss in central serous chorioretinopathy. Am J Ophthalmol 139:87-99

26. Moon JW, Yu HG, Kim TW, Kim HC, Chung H (2009) Prognostic factors related to photodynamic therapy for central serous chorioretinopathy. Graefes Arch Clin Exp Ophthalmol 247:1315-1323
27. Kim YY, Flaxel CJ (2011) Factors influencing the visual acuity of chronic central serous chorioretinopathy. Korean J Ophthalmol 25:90-97

28. Ooto S, Hangai M, Sakamoto A, Tsujikawa A, Yamashiro K, Ojima Y, Yamada Y, Mukai H, Oshima S, Inoue T, Yoshimura N (2010) High-resolution imaging of resolved central serous chorioretinopathy using adaptive optics scanning laser ophthalmoscopy. Ophthalmology 117(9):1800-1809

29. Fujita K, Yuzawa M, Mori R (2011) Retinal sensitivity after photodynamic therapy with half-dose verteporfin for chronic central serous chorioretinopathy: short-term results. Retina 31:772-778

30. Senturk F, Karacorlu M, Ozdemir H, Karacorlu SA, Uysal O (2011) Microperimetric changes after photodynamic therapy for central serous chorioretinopathy. Am J Ophthalmol 151:303-309

31. Reibaldi M, Boscia F, Avitabile T, Uva MG, Russo A, Zagari M, Occhipinti F, Russo V, Reibaldi A, Longo A (2011) Functional retinal changes measured by microperimetry in standard-fluence vs low-fluence photodynamic therapy in chronic central serous chorioretinopathy. Am J Ophthalmol 151:953-960

32. Ozdemir H, Senturk F, Karacorlu M, Karacorlu SA, Uysal O (2008) Macular sensitivity in eyes with central serous chorioretinopathy. Eur J Ophthalmol 18:799-804

33. Ozdemir H, Karacorlu SA, Senturk F, Karacorlu M, Uysal O (2008) Assessment of macular function by microperimetry in unilateral resolved central serous chorioretinopathy. Eye (Lond) 22:204-208

34. Ehrlich R, Mawer NP, Mody CH, Brand CS, Squirrell D (2012) Visual function following photodynamic therapy for central serous chorioretinopathy: a comparison of automated macular microperimetry versus best-corrected visual acuity. Clin Experiment Ophthalmol 40:e32-e39

35. Fujita K, Shinoda K, Imamura Y, Matsumoto CS, Mizutani Y, Mizota A, Yuzawa M (2012) Correlation of integrity of cone outer segment tips line with retinal sensitivity after half-dose photodynamic therapy for chronic central serous chorioretinopathy. Am J Ophthalmol 154(3):579-585

36. Ojima Y, Tsujikawa A, Hangai M, Nakanishi H, Inoue R, Sakamoto A, Yoshimura N (2008) Retinal sensitivity measured with the micro perimeter 1 after resolution of central serous chorioretinopathy. Am J Ophthalmol 146:77-84

37. Spaide RF, Koizumi H, Pozzoni MC (2008) Enhanced depth imaging spectral-domain optical coherence tomography. Am J Ophthalmol 146:496-500

38. Imamura Y, Fujiwara T, Margolis R, Spaide RF (2009) Enhanced depth imaging optical coherence tomography of the choroid in central serous chorioretinopathy. Retina 29:1469-1473

39. Maruko I, Iida T, Sugano Y, Ojima A, Sekiryu T (2011) Subfoveal choroidal thickness in fellow eyes of patients with central serous chorioretinopathy. Retina 31:1603-1608

40. Kim YT, Kang SW, Bai KH (2011) Choroidal thickness in both eyes of patients with unilaterally active central serous chorioretinopathy. Eye (Lond) 25:1635-1640

41. Maruko I, Iida T, Sugano Y, Ojima A, Ogasawara M, Spaide RF (2010) Subfoveal choroidal thickness after treatment of central serous chorioretinopathy. Ophthalmology 117:1792-1799

42. Maruko I, Iida T, Sugano Y, Furuta M, Sekiryu T (2011) One-year choroidal thickness results after photodynamic therapy for central serous chorioretinopathy. Retina 31:1921-1927 